\title{
Bisfenol (A) una toxina a tener en cuenta en el enfermo renal en hemodiálisis
}

\section{Bisphenol (A) uremic toxin to take into account in the Renal disease in Hemodialysis}

\author{
Enrique Bosch-Panadero ${ }^{1, *}$, Sebastián Mas Fontao ${ }^{2}$, Alberto Ruiz Priego 2 , \\ Jesús Egido ${ }^{3}$ Emilio González Parra ${ }^{4}$
}

${ }^{1} \mathrm{PhD}$, Laboratorio Renal, Vascular y Diabetes, Madrid, España

${ }^{2} \mathrm{PhD}$, Centro de Investigación Biomédica en Red en Diabetes y enfermedades metabólicas asociadas (CIBERDEM), Madrid, España

${ }^{3} \mathrm{MD}, \mathrm{PhD}$, Departamento de Nefrología e Hipertensión, Madrid, España, Red Española de Investigación Renal, Madrid, España

${ }^{4} \mathrm{MD}, \mathrm{PhD}$, Facultad de Medicina, Universidad Autónoma de Madrid, España; Fundación Renal Íñigo Álvarez de Toledo,

Instituto Reina Sofía de Investigación en Nefrología, Madrid, España

\begin{abstract}
Resumen
Muchas toxinas urémicas son originadas como consecuencia del catabolismo proteico por la flora intestinal. El metabolismo de aminoácidos aromáticos origina residuos de tipo fenólico. De estas toxinas, la más estudiada es el p-cresol, que se asocia a la función renal y daño vascular. El Bisfenol A (BPA) es una molécula exógena de características semejantes a estas toxinas urémicas aromáticas. El BPA es un disruptor endocrino estrogénico que se encuentra en latas de conserva, botellas de plástico, resinas epoxi y en algunos dializadores. Esta molécula se acumula en pacientes que tienen deteriorada la función renal. Estudios observacionales han demostrado que una exposición a BPA está vinculada, entre otras muchas, a lesión renal y cardiovascular en los seres humanos; en estudios en animales se ha descrito un vínculo causal. Los riñones con función renal normal excretan rápidamente BPA, pero una excreción insuficiente en pacientes con ERC da lugar a la acumulación del BPA en el organismo.
\end{abstract}

Palabras clave: toxina urémica, bisfenol A, enfermedad crónica renal, disruptor endocrino, hemodiálisis.

http://doi.org/10.22265/acnef.4.1.256

\begin{abstract}
Introduction: Most uremic toxins are by-products of protein metabolism by action of intestinal flora. The metabolism of aromatic amino acids originates phenolic type residues. The most studied is p-cresol that is associated with renal function and vascular damage. Bisphenol A (BPA) is an exogenous molecule with characteristics similar to these aromatic uremic toxins. BPA is an estrogenic endocrine disruptor, found in tin cans, plastic bottles, epoxy resins and in some dialyzers. This molecule accumulates in patients who have impaired renal function. Observational studies have shown that exposure of BPA is linked to renal and cardiovascular injury, among many others in humans, and in animal studies a causal link has been described. Kidneys with normal renal function rapidly excrete BPA, but insufficient excretion in patients with CKD results in accumulation of BPA in the body.
\end{abstract}

Key words: Uremic toxin, Bisphenol A, chronic kidney disease, endocrine disruptor, hemodialysis.

http://doi.org/10.22265/acnef.4.1.256

Referenciar este artículo: Bosch-Panadero E, Mas Fontao S, Ruiz Priego A, Egido J, González Parra E. Bisfenol (A) una toxina a tener en cuenta en el enfermo renal en hemodiálisis. Rev. Colomb. Nefrol. 2017;4(1): 57 - 68.

Correspondencia: *Enrique Bosch-Panadero, egparra@fjd.es

Recibido: 30-01-17 • Aceptado: 15-03-17 • Publicado en línea: 15-03-17. 


\section{Introducción}

$\mathrm{E}$ n España aproximadamente 4 millones de personas padecen enfermedad renal crónica (ERC), de las cuales unas 51.000 están en tratamiento renal sustitutivo, la mitad en diálisis y el resto con un trasplante renal funcional (Informe de Diálisis y Trasplante 2012). Cada año unas 6.000 personas con insuficiencia renal progresan en su enfermedad hasta llegar a la necesidad de seguir tratamiento sustitutivo renal. La prevalencia de la ERC aumenta de forma progresiva con el envejecimiento y con otras enfermedades como la diabetes tipo 2, la hipertensión arterial y la arterioesclerosis ${ }^{1}$. En la ERC se acumulan múltiples moléculas, denominadas toxinas urémicas, que son responsables de la sintomatología asociada y que contribuyen a un incremento de la morbilidad y mortalidad.Es de esperarse, por tanto, que la eliminación de toxinas urémica se acompañede una mejora de la situación clínica.

Las toxinas urémicas han sido clasificadas, según el tamaño, en aquellas de pequeño tamaño, con un peso molecular inferior a $500 \mathrm{Da}$; de mediano tamaño, con un peso molecular entre 500 y 5000 $\mathrm{Da}$; y las de gran tamaño, mayores de $5000 \mathrm{Da}^{2}$. Sin embargo, independientemente de su tamaño, muchas de ellas se encuentran unidas a proteínas, lo que dificulta su eliminación. Por ello, otra forma de clasificarlas se basa en su unión (o no) a proteínas. Entre las alteraciones directamente relacionadas con las toxinas urémicas, se encuentran la pérdida progresiva de la función renal, la morbimortalidad cardiovascular y la sintomatología urémica, tal como anorexia, vómitos, debilidad, trastornos del sueño y neuropatía, entre otros muchos.

Entre estas toxinas, existen algunas que, por tener un anillo aromático en su composición, son denominadas aromáticas (TUA). Particularmente relevantes en los enfermos renales son los productos originados por el metabolismo de la fenilalanina y la tirosina por las bacterias anaerobias intestinales, como son el p-cresol, el ácido fenilacético y fenoles; o los productos de degradación del triptófano que dan lugar, frecuentemente, a la formación de indoles ya que estos se acumulan en estos pacientes al no poder eliminarlo por la orina. Estos compuestos, tanto libres como en sus formas conjugadas en forma de sulfatos o glucuronatos, han sido ampliamente estudiados debido a su acumulación en los pacientes con insuficiencia renal. Existen otras toxinas con anillos aromáticos que no han sido estudiadas en relación con la ERC, como son 1-metoxiresorcinol, CMPF, hidroquinona, quinurenina, ácido quinurénico o 3-hidroxiquinurenona.

El bisfenol A (BPA) se encuentra entre las toxinas urémicas que se acumulan en el paciente renal y que pueden influir en su clínica. Es un estrógeno sintético que forma parte de múltiples plásticos usados comúnmente y forma parte del grupo de moléculas que se denominan disruptores endocrinos al ser capaces de alterar la homeostasis de procesos como la reproducción, el peso y el desarrollo. El BPA actúa como una hormona y puede alterar la función celular a concentraciones muy bajas. Los niveles de esta molécula aumentan en el paciente renal, pues su eliminación se da, fundamentalmente, por vía renal. El BPA es un tóxico ambiental que tiene anillos fenólicos y similitud estructural con los fenoles, aunque el origen de ambas moléculas es diferente. No obstante, el metabolismo y los efectos secundarios del BPA tienen características comunes con los fenoles de origen intestinal.

En la presente revisión analizamos el BPA como una toxina urémica de origen exógeno, englobada en el grupo de los fenoles. Aunque se trata de una molécula cuya toxicidad ha sido ampliamente estudiada, su uso no ha sido prohibido debido a que se elimina completamente por la orina. Sin embargo, la molécula en cuestión llama especialmente la atención del nefrólogo pues se relaciona con varias alteraciones renales y porque, al tener eliminación renal, se incrementa en los niveles sanguíneos y tisulares del paciente urémico. 


\section{Toxinas urémicas aromáticas}

El origen de las toxinas urémicas es múltiple. Algunas se originan en el metabolismo endógeno, otras en el metabolismo microbiano y otras, finalmente, proceden de una fuente exógena. La mayoría de las toxinas urémicas se originan en el metabolismo endógeno celular del individuo. Sin embargo, cada vez se conoce más la importancia de las toxinas generadas por el metabolismo microbiano intestinal ${ }^{3}$. También las toxinas procedentes de la dieta tienen un importante papel, como el oxalato y los productos avanzados de la glicoxilación ${ }^{4}$. Hay que mencionar que las toxinas urémicas se acumulan progresivamente a medida que la función renal decrece.

Los fenoles y los indoles son las toxinas urémicas unidas a proteínas más estudiadas. Se reconoce la implicación directa de ambas en la progresión de la insuficiencia renal y del daño vascular. Estas toxinas son metabolitos del catabolismo proteico por bacterias intestinales, el cual muestra un alto incremento en el paciente con ERC.

Diariamente alcanzan el intestino hasta $4 \mathrm{~g}$ de nitrógeno en forma de proteínas (50\%) y péptidos (20-30 \%). Estas proteínas son degradadas por proteasas y peptidasas a aminoácidos. Parte de estos alcanza el colon, donde es degradada por las bacterias intestinales dando origen a metabolitos potencialmente tóxicos como amonio, aminas, tioles, fenoles e indoles. Estos productos de la putrefacción en colon son eliminados casi totalmente por heces, aunque una parte es absorbida ${ }^{5}$.

Entre los fenoles se deben destacar el p-cresol y el p-cresilsulfato; el ácido fenilacético y el fenol ${ }^{6}$.

-P-Cresol, p-Cresilsulfato, y p-Cresol glucurónido: el p-cresol es un producto del metabolismo de la fenilalanina y la tirosina por parte de las bacterias anaerobias intestinales. La mayoría del p-cresol es conjugado por la flora en la pared intestinal a p-cresilsulfato y en el hígado a p-cresilglucurónido. La mayoría del p-cresol circula como p-cresilsulfato ${ }^{7}$, molécula que es un potente oxidante. El p-cresol libre (es decir, no unido a proteínas) está relacionado con daño cardiovascular en pacientes no diabéticos y con incremento de mortalidad cardiovascular ${ }^{8}$. Sin embargo, el método de medida del p-Cresol no distinguió el p-cresol como tal de la forma conjugada como p-cresilsulfato, de cuya toxicidad, además, existe evidencia ${ }^{6}$.

-El fenol procede fundamentalmente de la ingesta directa; del catabolismo de la tirosina y de otros sustratos por las bacterias intestinales; y del consumo de tabaco.

-El ácido fenilacético es el resultado de la degradación de la fenilalanina. Se une en un $30 \%$ de dicho acido a proteínas. Ha sido relacionado con alteración de la inmunoregulación en el paciente urémico, a la vez que participa en el estrés oxidativo ${ }^{9} \mathrm{e}$ inhibe la función del osteoblasto contribuyendo en las alteraciones del hueso ${ }^{10}$.

De los indoles debemos destacar el indoxilsulfato y el ácido indolacético ${ }^{6}$. El indol es una estructura aromática heterocíclica que se encuentra en muchos compuestos orgánicos como el triptófano y sus metabolitos. Las bacterias intestinales, al degradar triptófano, generan indol y ácido indolacético. El indol es posteriormente sulfatado por las enzimas hepáticas a indolsulfato. Hay que mencionar que estos metabolitos aumentan en casos de insuficiencia renal.

-Indoxilsulfato: es el más estudiado de los indoles y es una toxina renal y vascular. En hemodiálisis se asocia a ateroesclerosis ${ }^{11}$, a calcificación vascular y a disfunción endotelial ${ }^{12}$. También ha sido implicado en la progresión de la enfermedad renal al deteriorar la capacidad antioxidante celular y al ser una molécula proinflamatoria y profibrótica ${ }^{13}$. 
-Ácido indolacético: se ha relacionado con progresión de la fibrosis intersticial renal ${ }^{14}$.

\section{Principales complicaciones derivadas de la acu- mulación de toxinas urémicas de origen intes- tinal}

Las toxinas urémicas derivadas de la putrefacción de las proteínas por parte de las bacterias del colon, como fenoles e indoles, han sido relacionadas con múltiples alteraciones clínicas que presenta el paciente con ERC. Entre todas ellas podríamos destacar:

a) Progresión de la insuficiencia renal crónica: tanto el indoxilsulfato como el p-cresil sulfato han sido relacionados con el deterioro de la función renal, ya que acelera la progresion de la enfermedad $^{15}$. En un estudio prospectivo con 268 pacientes con ERC en diversos estadios, las concentraciones de ambas moléculas fueron predictivas de la progresión de la enfermedad renal, independientemente de otros factores de riesgo cardiovascular ${ }^{16}$.

b) Complicaciones cardiovasculares: se ha observado una relación entre las concentraciones de indoxilsulfato y daño vascular, así como calcificación aórtica, lo que implica directamente a esta molécula en el daño cardiovascular del paciente urémi$\mathrm{co}^{17}$. El indoxilsulfato está implicado, también, en el estrés oxidativo de las células endoteliales y en la proliferación de las células musculares lisas vasculares $^{18}$. Además, tiene un efecto profibrótico y aumenta la hipertrofia de los fibroblastos cardiacos ${ }^{19}$.

c) Anemia: el indoxilsulfato ha sido relacionado con la anemia del paciente renal al interferir con la adecuada producción de eritropoyetina ${ }^{20}$.

d) Osteodistrofia: el indoxilsulfato tiene efectos adversos sobre la formación ósea al promover estrés oxidativo en los osteoblastos e inducir resistencia a la PTH, con el desarrollo de un hueso adinámico ${ }^{21}$.

\section{Bisfenol A como toxina urémica}

\section{Generalidades del BPA}

En los últimos años ha despertado gran atención por parte de la comunidad científica el efecto nocivo de un tóxico ambiental, de tipo fenólico, el bisfenol A (BPA). En particular, también ha generado el interés entre la comunidad nefrológica pues se le ha relacionado con alteraciones renales y endocrinas, pero principalmente porque su eliminación es renal $\mathrm{y}$, por lo tanto, los niveles sanguíneos y tisulares de este tóxico se incrementan en el paciente renal.

El BPA es un ingrediente del plástico policarbonato y de las resinas epoxi, a los que añade dureza, claridad y poco peso, mientras resiste bien la temperatura y la electricidad. El BPA fue sintetizado en la década de los treinta como estrógeno sintético ${ }^{22}$. La aparición del dietilbestrol, en la misma época, desplazó este tipo de investigaciones hasta que se descubrió que el BPA actuaba como estabilizante en la elaboración de policarbonatos plásticos ${ }^{23}$. Los policarbonatos plásticos se emplean en envases y contenedores de uso común en la industria alimentaria y en el hogar, como botellas de plástico, biberones, lentillas, dispositivos médicos, etc. Las resinas epoxi que contienen BPA se usan como recubrimiento en las latas de comidas y bebida. Sin embargo, debido al posible impacto sobre la salud, el recubrimiento de epoxi fue remplazado en Japón por un filme de poliéster ${ }^{24}$.También se usa el BPA en la síntesis de polisulfonas, en cetonas de poliéter, como antioxidante en algunos plastificantes y como un inhibidor de polimerización en el PVC.

El BPA es un caso especial de toxina urémica dado que su aporte es exógeno, a diferencia de las toxinas normalmente consideradas. Aunque es un compuesto ubicuo, se encuentra principalmente, como se ha mencionado, en botellas de plástico, biberones, lentillas o utensilios médicos (dializadores). Las resinas epoxi que contienen BPA se usan como recubrimiento de latas usadas en alimentación, aunque, según se dijo, existe una tendencia a 
su sustitución por poliésteres ${ }^{24}$. Ante lo extendido de su uso, las agencias regulatorias llevan muchos años debatiendo el riesgo potencial del BPA como estrógeno sintético de consumo cotidiano para la salud humana.

El BPA suele ser pasar al torrente circulatorio a través de la vía oral, usualmente acompañando a los productos contenidos en recipientes de plástico que lo incluyen entre sus componentes. Al igual que ocurre con los fenoles de origen intestinal, el BPA se conjuga en el intestino y el hígado con ácido glucurónico y se elimina en su práctica totalidad por medio de la orina ${ }^{25}$.

También puede existir exposición por vía no oral al BPA, por lo que también es considerado un tóxico de origen ambiental. Sin embargo, la continua exposición del público a esta toxina despierta mayor controversia debido a su función como agonista endocrino. Aunque el BPA está catalogado comúnmente como disruptor endocrino, las autoridades europeas y americanas consideran que debido a su rápida eliminación se lo puede considerar un compuesto relativamente seguro a pesar de las evidencias publicadas ${ }^{26}$.

\section{EI BPA en la enfermedad renal crónica}

Como se mencionó, el BPA en condiciones normales se metaboliza en el hígado y se elimina por la orina. Estudios revelan que la excreción urinaria de BPA puede ser utilizada como biomarcador para la enfermedad renal ${ }^{27}$, pues la excreción urinaria de BPA disminuye con el deterioro de la función renal y estas asociaciones difieren según la edad o el sexo. El estudio también revela que existe una correlación entre la excreción de BPA y filtrado glomerular $(\mathrm{FG})^{28-30}$.

Los estudios publicados sobre BPA y pacientes con ERC observan que hay un aumento de BPA en sangre. Pacientes con una disminución de la función renal presentan un aumento de BPA en suero $\mathrm{y}$ en pacientes en hemodiálisis los niveles fueron todavía más altos ${ }^{31}$. Uno de los argumentos usado por los organismos gubernamentales para considerar seguro el uso de BPA en la población general es la eliminación urinaria casi completa de la molécula conjugada $^{32}$, lo que disminuye los riesgos de la exposición al BPA. Por todo lo anteriormente mencionado, los pacientes con enfermedad renal son objeto especial de estudio ya que son más sensibles a la acumulación de BPA y su toxicidad potencial debido a la pérdida de los mecanismos fisiológicos de excreción de BPA en orina.

Kretier y colegas observaron que a medida que desciende el FG aumentan los niveles séricos de $\mathrm{BPA}^{33}$.En nuestro estudio demostramos que los niveles séricos de BPA en pacientes en diálisis sin función renal residual son muy superiores a pacientes control con función renal residual. Esto confirma los estudios previos que observan que la incapacidad para excretar BPA por orina redunda en un aumento de los valores séricos de $\mathrm{BPA}^{33}$. Kanno et al. observaron que en los pacientes en hemodiálisis la concentración de BPA $(5,3 \pm 0,3 \mathrm{ng} / \mathrm{mL})$ es mayor que en pacientes en diálisis peritoneal $(3,8 \pm$ $0,2 \mathrm{ng} / \mathrm{mL}$ ) y mayor que en personas con función renal normal $(2,6 \pm 0,1 \mathrm{ng} / \mathrm{mL})^{34}$. El paciente renal no debería, entonces, estar incluido en la disposición general según la cual el BPA no es tóxico al eliminarse por el riñón, pues tiene alterada esta vía de eliminación. Por tanto, estaría de acuerdo con las recomendaciones cuidar la exposición al BPA de pacientes con $\mathrm{ERC}^{35}$. Sin embargo, como ya hemos comentado, en este grupo estaría un $10 \%$ de la población española, buena parte de la cual desconoce presentar la enfermedad y, por lo tanto, acumula el BPA en sangre y, posiblemente, en tejidos.

Entre los indicios que apuntan a un papel causal del BPA en la enfermedad renal, es necesario considerar que, en adultos sanos, niveles de BPA urinario $>1,4 \mu \mathrm{g} / \mathrm{L}$ se asocian con un $23 \%$ mayor de riesgo de microalbuminuria que en adultos y niños con niveles $<0,5 \mu \mathrm{g} / \mathrm{L} 29$. Asimismo, se ha relacionado con la existencia de albuminuria de bajo grado en adultos chinos ${ }^{36}$. Se han identificado po- 
sibles mecanismos de nefrotoxicidad mediada por BPA, relacionados con un aumento del estrés oxidativo, inflamación e inducción de la hipertensión arterial ${ }^{37,38}$. Además, recientemente el BPA sérico ha demostrado ser un predictor de progresión de enfermedad renal en pacientes con diabetes tipo $\mathrm{II}^{39}$, en el sentido de que pacientes con niveles más altos de BPA presentan mayor progresión de su enfermedad renal. Se ha demostrado que el BPA reduce la actividad antioxidante, lo que debe contribuir al estrés oxidativo19, también que el BPA reduce la viabilidad podocitaria37 y que es capaz de producir hipertrofia podocitaria, pues involucra a TFG- $\beta$ y la ciclina kinasa inhibidor p27kip1, el cual es conocido por su papel en el mecanismo de la hipertrofia de la célula renal ${ }^{40-42}$.

Sin embargo, el acúmulo de esta sustancia en el paciente con un descenso del filtrado glomerular ha motivado el análisis de la misma como posible toxina urémica. Entre los argumentos aducidos por los diferentes organismos oficiales para considerar seguro el uso del BPA en el consumo ordinario está, como se ha mencionado, la eliminación casi completa por orina de la molécula conjugada ${ }^{32}$. Por ese motivo, los pacientes con daño renal, en los que la eliminación de la molécula se encuentra alterada, deben considerarse una población especialmente sensible al BPA.

A mayor progresión de la enfermedad renal este bucle de retroalimentación aumentaría, en la medida que los pacientes con ERC no son capaces de excretar completamente el BPA en la orina ${ }^{43}$, lo que puede influir en el deterioro de la función renal ${ }^{31}$. Sin embargo, el National Health and Nutrition Examination Survey 2003-6 (NHANES III), sobre una muestra de 2573 pacientes, observó también descenso de eliminación de BPA con deterioro de la función renal, aunque este caso solo resultó significativo en mujeres ${ }^{29}$. Por su parte, en el estudio de Krieter se observa una correlación entre el deterioro de la función renal y las concentraciones plasmáticas de $\mathrm{BPA}^{33}$.

\section{Bisfenol A en diálisis}

El paciente en diálisis es un paciente con especial riesgo en la toxicidad debida al BPA, pues tiene completamente abolida la eliminación renal. Adicionalmente, en los pacientes en hemodiálisis se añade que el BPA, al ser un componente ubicuo, forma parte de la composición del material plástico de algunos dializadores y líneas de uso común, tanto en forma de policarbonato en las carcasas, como en múltiples membranas de diálisis, como es el caso de la polisulfona (PS) o el Polyester-Polymer alloy (PEPA).

En estos dializadores el polímero se halla en constante contacto con la sangre, lo que daría lugar a liberación del mismo hacia el torrente circulatorio. Por esta causa, el incremento de la toxina en el paciente en hemodiálisis no solo es debido a una exposición ambiental, sino posiblemente a la propia técnica empleada en el procedimiento ${ }^{44}$. Diferentes estudios han descrito que los efluentes de los dializadores compuestos por esos materiales tienen concentraciones elevadas ${ }^{31}$.

H. Shintani et al. analizaron cuatro tipos de dializadores de polisulfona presentes en el mercado, con carcasa de policarbonato o de copolímero de butadieno-poliestireno y con esterilización con vapor de agua o rayos gamma. El BPA aumenta más en dializadores con carcasa de policarbonato, independientemente de la membrana. Los dializadores de polisulfona con carcasa de policarbonato esterilizados con vapor de agua son los que mayor nivel de BPA se detecta en el efluente ${ }^{45}$. Conclusiones similares han sido encontradas por otros autores ${ }^{46}$.

Estos estudios muestran que la migración de BPA en los dializadores cuando se usa sangre es significativamente mayor que cuando se emplea salino salino. Posiblemente, esta diferencia se debe al efecto de componentes hidrofóbicos presentes en la sangre, como lípidos y lipoproteínas. Así, la concentración de BPA en el efluente de un líquido sobre 
una membrana de polisulfona es diferente si el flujo sobre ésta es de agua o de suero bobino (eluyéndose $3,78-141,8 \mathrm{ng}$ vs. $140.7-2090 \mathrm{mg}$ por dializador $)^{44}$.

El flujo también afectaría la cantidad de BPA extraída, debido al tiempo de residencia en el dializador. Por todo ello, aunque resulta muy difícil determinar en estos pacientes si las concentraciones plasmáticas superan las recomendadas, la mayoría de autores recomiendan usar en los pacientes en diálisis dializadores con un aporte inferior de BPA $\mathrm{y}$, además, intentar evitar materiales plásticos ricos en BPA, como botellas.

No existen estudios bien diseñados sobre la implicación de técnicas con gran trasporte convectivo, como la hemodiafiltración. Si bien es una técnica que puede depurar el BPA, no es menos cierto que se usa un líquido de reinfusión que atraviesa membranas con BPA, por lo que se podría estar incrementando su infusión. Será necesario determinar su implicación en estudios prospectivos y cruzados para observar qué ocurre en esta modalidad de tratamiento.

Por el contrario, en DP tan solo existe un estudio acerca de impacto del BPA, encontrándose que su concentración en el líquido de diálisis es muy inferior al nivel permitido. Aunque solo lo estudian en 4 pacientes, solo parece aumentar las concentraciones de BPA en un paciente. Por ello, los autores concluyen que el BPA no parece depurarse ni incrementar con la $\mathrm{DP}^{47}$.

Las evidencias recientemente halladas llevaron a que el Comité científico de la Unión Europea (Scientific Committee on Emerging and Newly Identified Health Risks) emitiese, a principios de 2015, un informe titulado "Final opinion on The safety of the use of bisphenol A in medical devices" en el que concluyen que existe un riesgo de efectos adversos derivados del BPA cuando está disponible por vía sistémica mediante rutas de exposición no oral, es- pecialmente para neonatos en unidades de cuidados intensivos, niños sometidos a procedimientos médicos prolongados o pacientes en diálisis ${ }^{48}$.

A pesar de esta certidumbre, prácticamente no existen estudios prospectivos a largo plazo que relacionen el efecto de la hemodiálisis con la presencia de BPA y sus posibles efectos. En 2013, Krieter et al estudiaron el efecto de la hemodiálisis en un periodo de 4 semanas, concluyendo que las diferencias entre las membranas con bisfenol (polisulfona) y sin bisfenol (polinefrona) no eran significativas 33 . Sin embargo, el hecho de que las carcasas de todos los dializadores empleados contuvieran BPA y el corto tiempo considerado por el estudio podrían explicar estos resultados.

Nuestro grupo, por su parte, utilizó un diseño prospectivo y cruzado, intercambiando dializadores con BPA (polisulfona) y sin BPA (polinefrona) durante 3 meses cada uno. Los niveles promedio de BPA aumentaron después de una sola sesión utilizando dializadores con polisulfona, mientras que no variaron en aquellos dializados con polinefrona. También el uso crónico de dializadores con membrana de polisulfona provocó un incremento de los niveles séricos de BPA, que no se observa en polinefrona ${ }^{49}$.Los niveles promedio de BPA aumentaron después de una sola sesión utilizando dializadores con polisulfona, mientras que no variaron en aquellos dializados con polinefrona. También el uso crónico de dializadores con membrana de polisulfona provocó un incremento de los niveles séricos de BPA que no se observa en polinefrona.

La determinación de BPA en células circulantes dio lugar a resultados semejantes y, en el periodo estudiado con dializadores con polisulfona, dio lugar a un aumento, mientras que en el caso de la polinefrona disminuyeron los niveles de BPA en un periodo de tiempo de 3 meses. Este incremento fue asociado tanto a un aumento de los niveles de radicales libres intracelulares y marcadores inflamatorios circulantes (IL-6, TNF- $\alpha$, proteína $\mathrm{C}$ reactiva) 
en los pacientes, como en ensayos in vitro utilizando células circulantes, en los que se observó que las membranas de polisulfona liberan bisfenol al medio y esto da lugar a un aumento de producción de citoquinas en linfocitos en cultivo.

Este es el primer estudio prospectivo realizado con el fin de evaluar el impacto de los dializadores libres de BPA, por lo que se requieren estudios prospectivos para evaluar si los incrementos observados de BPA y sus efectos en aumento del estrés oxidativo o inflamatorio se asocian a un peor pronóstico clínico en estos pacientes.

En suma, el bisfenol A es un estrógeno sintético que se encuentra formando parte de plásticos de uso cotidiano y material de hemodiálisis, como algunas membranas. Se elimina por el riñón, por lo que se acumula en el enfermo renal. Su molécula es similar a otras toxinas urémicas con anillo aromático, como el pCresol, muy estudiadas por tener un papel significativo en la morbimortalidad del paciente renal. El metabolismo y relación con inflamación y oxidación son muy similares entre ambas. Aunque quedan preguntas pendientes, como conocer la concentración tisular de BPA en el paciente en hemodiálisis, existen evidencias para evitar dentro de lo posible el bisfenol A en estos pacientes. El informe de expertos de la Unión Europea, plasmado en el informe del SCENIHR, defiende esta postura ${ }^{48,50}$. Consideramos, por lo tanto, que el bisfenol A debe de ser considerado una toxina urémica de origen exógeno.

\section{Agradecimientos}

Este trabajo fue apoyado por una subvención de FIS PI16 / 01298, Sociedad Madrileña de Nefrología (SOMANE), Fundación Renal Íñigo Álvarez de Toledo (FRIAT).

\section{Conflicto de intereses}

El Laboratorio de nefrología ha recibido una beca de Nipro Corporation para investigar los efectos de bisfenol en los pacientes renales. Sin embargo, esta empresa no ha participado en el diseño del estudio, interpretación de los resultados ni escritura del manuscrito.

\section{Responsabilidades éticas}

\section{Protección de personas y animales}

Los autores declaran que para esta investigación no se han realizado experimentos en seres humanos ni en animales.

\section{Confidencialidad de los datos}

Los autores declaran que en este artículo no aparecen datos de pacientes.

\section{Derecho a la privacidad y consentimiento informado}

Los autores declaran que en este artículo no aparecen datos de pacientes. 


\section{Referencias}

1. Shlipak MG, Fried LF, Cushman M, Manolio TA, Peterson D, Stehman-Breen C, et al. Cardiovascular Mortality Risk in Chronic Kidney Disease. JAMA[Internet]. 2005 [accedido 01 Jan 2000];293(14):1737. Disponible en: https://doi.org/10.1001/jama.293.14.1737

2. Vanholder R, Baurmeister U, Brunet P, Cohen G, Glorieux G, Jankowski J. A bench to bedside view of uremic toxins. J Am Soc Nephrol [Internet]. 2008 [accedido 01 Jan 2000];19(5):863-70. Disponible en: https://doi.org/10.1681/ASN.2007121377

3. Wikoff WR, Anfora AT, Liu J, Schultz PG, Lesley SA, Peters EC, et al. Metabolomics analysis reveals large effects of gut microflora on mammalian blood metabolites. Proc Natl Acad Sci U S A [Internet]. 2009 [accedido 01 Jan 2000];106(10):3698-703. Disponible en: https://doi.org/10.1073/ pnas.0812874106

4. Goldfarb DS, Modersitzki F, Asplin JR. A randomized, controlled trial of lactic acid bacteria for idiopathic hyperoxaluria. Clin J Am Soc Nephrol [Internet]. 2007 [accedido 01 Jan 2000];2(4):745-9. Disponible en: https://doi.org/10.2215/CJN.00600207

5. Bammens B, Evenepoel P, Verbeke K, Vanrenterghem Y. Removal of the protein-bound solute p-cresol by convective transport: a randomized crossover study. Am J Kidney Dis [Internet]. 2004 [accedido 01 Jan 2000]; 44(2):278-85. Disponible en: https://doi.org/10.1053/j.ajkd.2004.04.033

6. Jourde-Chiche N, Dou L, Cerini C, Dignat-George F, Vanholder R, Brunet P. Progress in Uremic Toxic Research: Protein-bound toxins-Update 2009. Semin Dial [Internet]. 2009 [accedido 01 Jan 2000];22(4):334-9. Disponible en: https://doi.org/10.1111/j.1525-139X.2009.00576.x

7. Martinez AW, Recht NS, Hostetter TH, Meyer TW. Removal of P-cresol sulfate by hemodialysis. J Am Soc Nephrol [Internet]. 2005 [accedido 01 Jan 2000];16(11):3430-6. Disponible en: https://doi. org/10.1681/ASN.2005030310

8. Meijers BKI, Bammens B, De Moor B, Verbeke K, Vanrenterghem Y, Evenepoel P. Free p-cresol is associated with cardiovascular disease in hemodialysis patients. Kidney Int [Internet]. 2008 [accedido 01 Jan 2000];73(10):1174-80. Disponible en: https://doi.org/10.1038/ki.2008.31

9. Schmidt S, Westhoff TH, Krauser P, Zidek W, van der Giet M. The uraemic toxin phenylacetic acid increases the formation of reactive oxygen species in vascular smooth muscle cells. Nephrol Dial Transplant [Internet]. 2008 [accedido 01 Jan 2000];23(1):65-71. Disponible en: https://doi.org/10.1093/ndt/ gfm475

10. Yano S, Yamaguchi T, Kanazawa I, Ogawa N, Hayashi K, Yamauchi M, et al. The uraemic toxin phenylacetic acid inhibits osteoblastic proliferation and differentiation: an implication for the pathogenesis of low turnover bone in chronic renal failure. Nephrol Dial Transplant [Internet]. 2007 [accedido 01 Jan 2000];22(11):3160-5. Disponible en: https://doi.org/10.1093/ndt/gfm455

11. Raff AC, Meyer TW, Hostetter TH. New insights into uremic toxicity. Curr Opin Nephrol Hypertens [Internet]. 2008 [accedido 01 Jun 2000];17(6):560-5. Disponible en: https://doi.org/10.1097/MNH. 0b013e32830f45b6

12. Faure V, Dou L, Sabatier F, Cerini C, Sampol J, Berland Y, et al. Elevation of circulating endothelial microparticles in patients with chronic renal failure. J Thromb Haemost [Internet]. 2006 [accedido 01 Jun 2000];4(3):566-73. Disponible en: https://doi.org/10.1111/j.1538-7836.2005.01780.x

13. Motojima M, Hosokawa A, Yamato H, Muraki T, Yoshioka T. Uremic toxins of organic anions up-regulate PAI-1 expression by induction of NF-kappaB and free radical in proximal tubular cells. Kidney 
Int [Internet]. 2003 [accedido 01 Jun 2000];63(5):1671-80. Disponible en: https://doi.org/10.1046/ j.1523-1755.2003.00906.x

14. Satoh M, Hayashi H, Watanabe M, Ueda K, Yamato H, Yoshioka T, et al. Uremic toxins overload accelerates renal damage in a rat model of chronic renal failure. Nephron Exp Nephrol[Internet]. 2003 [accedido 01 Jun 2000];95(3):e111-8. Disponible en: https://doi.org/10.1159/000074327

15. Meijers BKI, Evenepoel P. The gut-kidney axis: indoxyl sulfate, p-cresyl sulfate and CKD progression. Nephrol Dial Transplant [Internet]. 2011 [accedido 01 Jun 2000];26(3):759-61. Disponible en: https://doi.org/10.1093/ndt/gfq818

16. Wu I-W, Hsu K-H, Lee C-C, Sun C-Y, Hsu H-J, Tsai C-J, et al. p-Cresyl sulphate and indoxyl sulphate predict progression of chronic kidney disease. Nephrol Dial Transplant [Internet]. 2011 [accedido 01 Jun 2000];26(3):938-47. Disponible en: https://doi.org/10.1093/ndt/gfq580

17. Barreto FC, Barreto DV, Liabeuf S, Meert N, Glorieux G, Temmar M, et al. Serum indoxyl sulfate is associated with vascular disease and mortality in chronic kidney disease patients. Clin J Am Soc Nephrol [Internet]. 2009 [accedido 01 Jun 2000];4(10):1551-8. Disponible en: https://doi.org/10.2215/ CJN.03980609

18. Yamamoto $\mathrm{H}$, Tsuruoka $\mathrm{S}$, Ioka $\mathrm{T}$, Ando $\mathrm{H}$, Ito $\mathrm{C}$, Akimoto $\mathrm{T}$, et al. Indoxyl sulfate stimulates proliferation of rat vascular smooth muscle cells. Kidney Int [Internet]. 2006 [accedido 01 Jun 2000];69(10):1780-5. Disponible en: https://doi.org/10.1038/sj.ki.5000340

19. Lekawanvijit S, Adrahtas A, Kelly DJ, Kompa AR, Wang BH, Krum H. Does indoxyl sulfate, a uraemic toxin, have direct effects on cardiac fibroblasts and myocytes? Eur Heart J [Internet]. 2010 [accedido 01 Jun 2000];31(14):1771-9. Disponible en: https://doi.org/10.1093/eurheartj/ehp574

20. Chiang C-K, Tanaka T, Inagi R, Fujita T, Nangaku M. Indoxyl sulfate, a representative uremic toxin, suppresses erythropoietin production in a HIF-dependent manner. Lab Invest [Internet]. 2011[accedido 01 Jun 2000];91(11):1564-71. Disponible en: https://doi.org/10.1038/labinvest.2011.114

21. Nii-Kono T, Iwasaki Y, Uchida M, Fujieda A, Hosokawa A, Motojima M, et al. Indoxyl sulfate induces skeletal resistance to parathyroid hormone in cultured osteoblastic cells. Kidney Int [Internet]. 2007 [accedido 01 Jun 2000];71(8):738-43. Disponible en: https://doi.org/10.1038/sj.ki.5002097

22. Dodds EC, Goldberg L, Lawson W, Robinson R. OEstrogenic Activity of Certain Synthetic Compounds. Nature [Internet].1938 [accedido 01 Jun 2000];141:247-8. Disponible en: https://doi.or$\mathrm{g} / 10.1038 / 141247 \mathrm{~b} 0$

23. Casajuana N, Lacorte S. New methodology for the determination of phthalate esters, bisphenol A, bisphenol A diglycidyl ether, and nonylphenol in commercial whole milk samples. J Agric Food Chem [Internet]. 2004 [accedido 01 Jun 2000];52(12):3702-7. Disponible en: https://doi.org/10.1021/ jf040027s

24. Thomson BM, Grounds PR. Bisphenol A in canned foods in New Zealand: an exposure assessment. Food Addit Contam [Internet]. 2005 [accedido 01 Jun 2000];22(1):65-72. Disponible en: https://doi. org/10.1080/02652030400027920

25. Dekant W, Volkel W. Human exposure to bisphenol A by biomonitoring: methods, results and assessment of environmental exposures. Toxicol Appl Pharmacol [Internet]. 2008 [accedido 01 Jun 2000];228(1):114-34. Disponible en: https://doi.org/10.1016/j.taap.2007.12.008

26. U.S. Food and Drug Administration. Draft Assessment of Bisphenol A for Use in Food Contract Applications [Internet]. 2008 [accedido 01 Jun 2000]:1-105. Disponible en: https://www.fda.gov/ohr- 
ms/dockets/ac/08/briefing/2008-0038b1_01_02_FDA\%20BPA\%20Draft\%20Assessment.pdf

27. Davidson I, Gallieni M, Saxena R, Dolmatch B. A patient centered decision making dialysis access algorithm. J Vasc Access [Internet]. 2007 [accedido 01 Jun 2000];8(2):59-68. Disponible en: https:// www.ncbi.nlm.nih.gov/pubmed/17534790

28. Kataria A, Trasande L, Trachtman H. The effects of environmental chemicals on renal function. Nat Rev Nephrol [Internet]. 2015 [accedido 01 Jun 2000];11(10):610-25. Disponible en: https://doi. org/10.1038/nrneph.2015.94

29. Calafat AM, Kuklenyik Z, Reidy JA, Caudill SP, Ekong J, Needham LL. Urinary concentrations of bisphenol A and 4-nonylphenol in a human reference population. Environ Health Perspect [Internet]. 2005 [accedido 01 Jun 2000];113(4):391-5. Disponible en: https://doi.org/10.1289/ehp.7534

30. Cutanda F, Koch HM, Esteban M, Sanchez J, Angerer J, Castano A. Urinary levels of eight phthalate metabolites and bisphenol A in mother-child pairs from two Spanish locations. Int J Hyg Environ Health [Internet]. 2015 [accedido 01 Jun 2000];218(1):47-57. Disponible en: https://doi.org/10.1016/j. ijheh.2014.07.005

31. Murakami K, Ohashi A, Hori H, Hibiya M, Shoji Y, Kunisaki M, et al. Accumulation of bisphenol A in hemodialysis patients. Blood Purif [Internet]. 2007 [accedido 01 Jun 2000];25(3):290-4. Disponible en: https://doi.org/10.1159/000104869

32. Boeniger MF, Lowry LK, Rosenberg J. Interpretation of urine results used to assess chemical exposure with emphasis on creatinine adjustments: a review. Am Ind Hyg Assoc J [Internet]. 1993 [accedido 01 Jun 2000]; 54(10):615-27. Disponible en: https://doi.org/10.1080/15298669391355134

33. Krieter DH, Canaud B, Lemke H-D, Rodriguez A, Morgenroth A, von Appen K, et al. Bisphenol A in chronic kidney disease. Artif Organs [Internet]. 2013 [accedido 01 Jun 2000];37(3):283-90. Disponible en: https://doi.org/10.1111/j.1525-1594.2012.01556.x

34. Kanno Y, Okada H, Kobayashi T, Takenaka T, Suzuki H. Effects of endocrine disrupting substance on estrogen receptor gene transcription in dialysis patients. Ther Apher Dial [Internet]. 2007 [accedido 01 Jun 2000]; 11(4):262-5. Disponible en: https://doi.org/10.1111/j.1744-9987.2007.00472.x

35. You L, Zhu X, Shrubsole MJ, Fan H, Chen J, Dong J, et al. Renal function, bisphenol A, and alkylphenols: results from the National Health and Nutrition Examination Survey (NHANES 2003-2006). Environ Health Perspect [Internet]. 2011 [accedido 01 Jun 2000];119(4):527-33. Disponible en: https:// doi.org/10.1289/ehp.1002572

36. Li M, Bi Y, Qi L, Wang T, Xu M, Huang Y, et al. Exposure to bisphenol A is associated with low-grade albuminuria in Chinese adults. Kidney Int [Internet]. 2012 [accedido 01 Jun 2000];81(11):1131-9. Disponible en: https://doi.org/10.1038/ki.2012.6

37. Olea-Herrero N, Arenas MI, Munoz-Moreno C, Moreno-Gomez-Toledano R, Gonzalez-Santander M, Arribas I, et al. Bisphenol-A induces podocytopathy with proteinuria in mice. J Cell Physiol [Internet]. 2014 [accedido 01 Jun 2000];229(12):2057-66. Disponible en: https://doi.org/10.1002/jcp.24665

38. Saura M, Marquez S, Reventun P, Olea-Herrero N, Arenas MI, Moreno-Gomez-Toledano R, et al. Oral administration of bisphenol A induces high blood pressure through angiotensin II/CaMKII-dependent uncoupling of eNOS. Faseb J [Internet]. 2014 [accedido 01 Jun 2000];28(11):4719-28. Disponible en: https://doi.org/10.1096/fj.14-252460 
39. Hu J, Yang S, Wang Y, Goswami R, Peng C, Gao R, et al. Serum bisphenol A and progression of type 2 diabetic nephropathy: a 6-year prospective study. Acta Diabetol [Internet]. 2015 [accedido 01 Jun 2000];52(6):1135-41. Disponible en: https://doi.org/10.1007/s00592-015-0801-5

40. Romero M, Ortega A, Izquierdo A, Lopez-Luna P, Bosch RJ. Parathyroid hormone-related protein induces hypertrophy in podocytes via TGF-beta(1) and p27(Kip1): implications for diabetic nephropathy. Nephrol Dial Transplant [Internet]. 2010 [accedido 01 Jun 2000]; 25(8):2447-57. Disponible en: https://doi.org/10.1093/ndt/gfq104

41. Iglesias-de la Cruz MC, Ziyadeh FN, Isono M, Kouahou M, Han DC, Kalluri R, et al. Effects of high glucose and TGF-beta1 on the expression of collagen IV and vascular endothelial growth factor in mouse podocytes. Kidney Int [Internet]. 2002 [accedido 01 Jun 2000]; 62(3):901-13. Disponible en: https://doi.org/10.1046/j.1523-1755.2002.00528.x

42. Wolf G, Ziyadeh FN. Molecular mechanisms of diabetic renal hypertrophy. Kidney Int [Internet]. 1999 [accedido 01 Jun 2000]; 56(2):393-405. Disponible en: https://doi.org/10.1046/j.15231755.1999.00590.x

43. Hengstler JG, Foth H, Gebel T, Kramer P-J, Lilienblum W, Schweinfurth H, et al. Critical evaluation of key evidence on the human health hazards of exposure to bisphenol A. Crit Rev Toxicol [Internet]. 2011 [accedido 01 Jun 2000];41(4):263-91. Disponible en:https://doi.org/10.3109/10408444.2011.55 8487

44. Haishima Y, Hayashi Y, Yagami T, Nakamura A. Elution of bisphenol-A from hemodialyzers consisting of polycarbonate and polysulfone resins. J Biomed Mater Res [Internet]. 2001 [accedido 01 Jun 2000];58(2):209-15. Disponible en: https://doi.org/10.1002/1097-4636(2001)58:2<209::AID-JBM1009>3.0.CO;2-7

45. Shintani H. Determination of the endocrine disrupter bisphenol-A in the blood of uremia patients treated by dialysis. Chromatographia [Internet]. 2001 [accedido 01 Jun 2000];(53):331-3. Disponible en: https://doi.org/10.1007/BF02490435

46. Yamasaki H, Nagake Y, Makino H. Determination of bisphenol a in effluents of hemodialyzers. Nephron [Internet]. 2001 [accedido 01 Jun 2000];88(4):376-8. Disponible en: https://doi.org/10.1159/000046023

47. Sugimura K, Naganuma T, Kakiya Y, Okada C, Sugimura T, Kishimoto T. Endocrine-disrupting chemicals in CAPD dialysate and effluent. Blood Purif [Internet]. 2001 [accedido 01 Jun 2000];19(1):213. Disponible en: https://doi.org/10.1159/000014473

48. Scientific Committee on Emerging and Newly Identified Health Risk. Opinion on The safety of the use of bisphenol A in medical devices. 2015 [accedido 01 Jun 2000]. Disponible en: https://doi. org/10.2772/75546

49. Bosch-panadero E, Mas S, Sanchez-ospina D, Camarero V. The Choice of Hemodialysis Membrane Affects Serum Bisphenol A Levels. 2015;1-10.:

50. European Food Safety Authority. Bisphenol A [Internet]. [lugar desconocido]: European Food Safety Authority; 2015 [accedido 01 Jun 2000]. Disponible en: http://www.efsa.europa.eu/en/topics/topic/ bisphenol 